\title{
The String Theory Landscape
}

\author{
Michael R. Douglas
}

Simons Center for Geometry and Physics, Stony Brook University, Stony Brook, NY 11794, USA; mdouglas@scgp.stonybrook.edu

Received: 11 June 2019; Accepted: 18 July 2019; Published: 20 July 2019

\begin{abstract}
String/M theory is formulated in 10 and 11 space-time dimensions; in order to describe our universe, we must postulate that six or seven of the spatial dimensions form a small compact manifold. In 1985, Candelas et al. showed that by taking the extra dimensions to be a Calabi-Yau manifold, one could obtain the grand unified theories which had previously been postulated as extensions of the Standard Model of particle physics. Over the years since, many more such compactifications were found. In the early 2000s, progress in nonperturbative string theory enabled computing the approximate effective potential for many compactifications, and it was found that they have metastable local minima with small cosmological constant. Thus, string/M theory appears to have many vacuum configurations which could describe our universe. By combining results on these vacua with a measure factor derived using the theory of eternal inflation, one gets a theoretical framework which realizes earlier ideas about the multiverse, including the anthropic solution to the cosmological constant problem. We review these arguments and some of the criticisms, with their implications for the prediction of low energy supersymmetry and hidden matter sectors, as well as recent work on a variation on eternal inflation theory motivated by computational complexity considerations.
\end{abstract}

Keywords: string theory; quantum cosmology; string landscape

\section{Introduction}

Superstring theory and $\mathrm{M}$ theory are quantum theories of matter, gravity and gauge forces, in which the fundamental degrees of freedom are not particles but extended objects: one-dimensional strings and higher dimensional branes. This solves the problem of the nonrenormalizability of quantum gravity, at the cost of requiring the dimension of space-time to be ten (for superstrings) or eleven (for M theory). Nevertheless, by following the Kaluza-Klein approach of taking the extra dimensions to be a small compact manifold, one can argue that the resulting four dimensional theory can reproduce the Standard Model at low energies. This was first done by Candelas et al. in 1985 [1] and, ever since, superstring theory has been considered a leading candidate for a fundamental theory describing all physics in our universe.

In the years since, not only were the original arguments developed and sharpened, the most attractive competing candidate theories were shown to be equivalent to other regimes of string theory, obtained by taking the string coupling large or by taking the size of the extra dimensions to be sub-Planckian. In particular, eleven-dimensional supergravity, arguably the most symmetric extension of general relativity, turned out to be the strong coupling limit of type IIa superstring theory [2]. Conversely, $11 \mathrm{~d}$ supergravity contains a membrane solution, and one can obtain string theory from it by compactifying on a sub-Planckian circle, so that a membrane wound around the circle becomes a string. This larger picture containing both string theory and 11d supergravity is sometimes called $\mathrm{M}$ theory; we will call it string/M theory to emphasize that all of the superstring theories and $11 \mathrm{~d}$ supergravity are contained within this single framework. At present, it is the only theoretical framework that has been convincingly shown to quantize gravity in more than three space-time dimensions. 
In this brief review, we explain how string/M theory realizes the concept of a multiverse. The primary argument is to look at the construction of quasi-realistic four-dimensional compactifications (by which we mean those which are similar to the Standard Model but not necessarily agreeing with it in all detail) and enumerate the choices which enter this construction. This includes the choice of topology and geometry of the compactification manifold, the choice of auxiliary physical elements such as branes and generalized magnetic fluxes and how they are placed in the compact dimensions, and the choice of metastable minimum of the resulting effective potential. One can roughly estimate the number of choices at each step, and argue that they combine to produce a combinatorially large number of metastable vacua. These arguments are still in their early days and there is as yet no consensus on the number; estimates range from $10^{500}$ [3] which at the time it was made seemed large, to the recent $10^{272,000}[4]$.

Any of these compactifications are a priori candidates to describe the observed universe. Having chosen one, the next step in analyzing it is to compute or at least estimate the effective potential. This is a function of the scalar fields or "moduli" which parameterize the Ricci flat metric and other fields in the extra dimensions, including the overall volume of the extra dimensions, the string coupling constant (or "dilaton") and typically hundreds or even thousands of additional fields. As in nongravitational physics, the effective potential has both classical contributions (for example, see Equation (5) below) and quantum contributions (Casimir energies, instanton effects, etc.), and must be computed by approximate methods. One then looks for its metastable minima and analyzes the small fluctuations around it, to get the four-dimensional particle spectrum and interactions. To be clear, the definition of "vacuum" in this review is a metastable minimum of the effective potential. ${ }^{1}$ This is to be distinguished from "universe," "pocket universe," or "bubble," terms which denote a causally connected region of the multiverse in which the compactification takes a particular size and shape, and which thus sits in a single vacuum. Many universes in a multiverse could sit in the same vacuum, and this is why cosmology will predict a nontrivial probability distribution over vacua.

The effective potential of a string/M theory compactification, while mathematically just a single real-valued function, is a very complex object that summarizes a vast range of possible physical structures of the vacua and phase transitions between them. The set of effective potentials for all the compactifications is clearly far more complex. While computing them is a tall order, the rich mathematical structure of string/M theory compactification has led to amazing progress in this endeavor. While this rapidly gets very technical, it is here that we see how important it is that solutions of string/M theory are mathematically natural and-yes, beautiful—constructs. Although this beauty is subjective and cannot be regarded as an argument for or against their relevance to nature, it is what allows us to compute their properties and get objective information we can use to judge this point. In addition, this study is in its early days; we can be confident that progress in classifying and computing the $\mathrm{ab}$ initio predictions of string/M theory vacua will continue.

There are far too many vacua to study each one individually. In studying the string landscape, the next step is to estimate the distribution of observables among the vacua, using statistical techniques surveyed in [5]. A particularly important example is the distribution of values of the effective potential at the minima, in other words the cosmological constant (or c.c.). This is an important input into the arguments for the multiverse from cosmology and especially for the anthropic solution to the cosmological constant problem, for which we refer to [6,7] and the article [8] in this issue. This argument requires the existence of a large number of vacua such that the a priori probability that we will observe a given vacuum is roughly uniform in the cosmological constant at small values.

The a priori probability for making a specified observation, say that the cosmological constant $\Lambda$ sits in a given range of values, is a central theoretical concept in quantum cosmology, usually called the "measure factor." At first, one might think to define it as a sum over each of the vacua $V_{i}$ which

1 We might impose additional physical conditions if convenient, such as positive cosmological constant. 
realize the specified physical observable, weighted by the probability $P(i)$ with which we as observers believe we live in a universe described by $V_{i}$. However, it is better to break down these probabilities further and write the measure as a product of three factors. The first is a sum over vacua, while the second "cosmological" factor, call this $U(i)$, is the expected number of universes in the multiverse which realize the vacuum $V_{i}$. The third "anthropic" factor, which we call $A(i)$, is the expected number of observers in a universe of type $i$. We can then write the expectation value of an observable $O$ as

$$
\mathbb{E}[O]=\frac{\sum_{i} U(i) A(i) O(i)}{\sum_{i} U(i) A(i)},
$$

where $O(i)$ is the value of the observable in the vacuum labelled $i$. Thus, the probabilities are

$$
P(i)=\frac{U(i) A(i)}{\sum_{i} U(i) A(i)} .
$$

A reason to distinguish these three factors is that they are governed by different laws and the problems of understanding them can to some extent be separated. The set of vacua $\left\{V_{i}\right\}$ and many of the corresponding observations $O(i)$ are "static" questions for the fundamental theory (say string/M theory) which could be answered without knowing how the vacua were created. As an example, in a string compactification realizing the Standard Model, we can compute the fine structure constant by combining ideas from Kaluza-Klein theory with quantum field theory and the renormalization group, without knowing its cosmological origins. Crucially, to compute $O(i)$, we only need information about the vacuum $V_{i}$, making such problems relatively accessible to present knowledge. $^{2}$

By contrast, the factors $U(i)$ summarize information about how vacua are created in quantum cosmology. As we will discuss in Section 3, this subject depends on sweeping and difficult to justify theoretical assumptions, and the analysis requires knowledge about all of the vacua. While we may be at the point of beginning to ask meaningful questions here, it will be some time before we can have any confidence in statements whose justification requires knowledge about all vacua.

Finally, the numbers $A(i)$ depend on a vast panoply of scientific theories, ranging from topics in cosmology such as baryogenesis and big bang nucleosynthesis, through the astrophysics of structure formation at many scales, to some of the deepest questions in biology and philosophy (what is an observer, anyways?). One would be tempted to dismiss the factor $A(i)$ as totally intractable, were it not that the anthropic solution to the cosmological constant problem requires us to discuss it. Fortunately, the more physical determinants of $A(i)$, such as the size of the universe, number of galaxies and stars and the like, can be estimated, as discussed in the article [9] in this issue.

Given a particular observable $O$, we can identify two limiting possibilities. One is that $P(i)$ is highly correlated with the value $O(i)$. In this case, clearly it is crucial to estimate $P(i)$ to compute $\mathbb{E}[O]$. The other extreme is when $P(i)$ is totally uncorrelated with $O(i)$, so that

$$
\sum_{i} P(i) O(i) \sim \frac{1}{N_{\text {vacua }}} \sum_{i} P(i) \times \sum_{i} O(i)
$$

Since $\sum_{i} P(i)=1$, this means that

$$
\mathbb{E}[O] \sim \frac{1}{N_{\text {vacua }}} \sum_{i} O(i)
$$

2 Some observables, for example $\delta \rho / \rho$, do depend on the early history of the universe. To discuss these we would need to generalize Equation (1), but we can still work in terms of the four dimensional degrees of freedom visible in the vacuum $V_{i}$. 
In other words, the probability that we observe a given value for $O$ will be proportional to the number of vacua that realize it. This is the vacuum counting measure, whose study was formalized in [10].

Almost all statistical studies of the string landscape to date study the vacuum counting measure. For it to make sense, $N_{\text {vacua }}$ must be finite. This was shown in [11] using ideas we review in Section 2.

One of the most important claims about the string vacuum counting measure is that it is roughly uniform in the cosmological constant near zero. This was first argued in a toy model by Bousso and Polchinski [12], and then in successively more realistic models $[3,13]$. Note, however, that it is not at all obvious that the factor $U(i)$ is uniform near $\Lambda=0$, as we will discuss in Section 3.

One can also study the counting distribution of other observables. An example of great interest is the distribution of the supersymmetry breaking scale, and the distribution of masses of superpartners. One of the primary motivations for building the Large Hadron Collider was the hope that supersymmetry will be discovered there, which is feasible if there are superpartners with mass up to about $1 \mathrm{TeV}$. In addition, the discovery of supersymmetry was widely predicted, based on the "naturalness" argument. This is the idea is that there should be an explanation within the fundamental theory for the small number $M_{\text {Higgs }} / M_{\text {Planck }} \sim 10^{-17}$. One might try to trace this to a small parameter in the fundamental theory, but since renormalization in quantum field theory affects the masses of scalars additively, $M^{2} \rightarrow M^{2}+c \Lambda^{2}$ in terms of the cutoff energy $\Lambda$ and some order one $c$, achieving the small observed Higgs mass requires not a small bare mass but rather a bare mass finely tuned to cancel the effects of renormalization. By the same arguments made for the cosmological constant [6,7], this is implausible, posing the "hierarchy problem." By showing that the bare Higgs mass squared is uniformly distributed in quasi-realistic vacua, one can quantify this implausibility: the fraction of vacua in which fine-tuning is needed to obtain the observed Higgs mass is about $10^{-34}$.

During the 1970s and 1980s, a major search was made for mechanisms to produce small $M_{\text {Higgs }}$ without fine-tuning, with the leading candidates being technicolor and dynamical supersymmetry breaking. Technicolor made other predictions, such as flavor changing neutral currents, which were falsified during the 1990s by precision experiments. This left low energy supersymmetry as the favored explanation. To realize small $M_{\text {Higgs }}$ without fine-tuning in this framework, one postulates an additional matter sector with an asymptotically free gauge group, such that supersymmetry is spontaneously broken at the energy scale where its gauge coupling is strong. One then arranges the total theory to mediate this supersymmetry breaking to the Standard Model, either through additional matter (gauge mediated supersymmetry breaking) or through gravitational couplings (gravity mediated). This mediation will generically give mass to all of the superpartners of the observed Standard Model particles, and can give mass to the Higgs boson(s) as well. In this way, one can get natural classes of models in which a large fraction of vacua have small $M_{\text {Higgs }}$. String compactifications in which all of these ingredients appear were constructed in many works—see, for example, [14-17] and the review [18].

Upon formulating the problem of the distribution of supersymmetry breaking scales systematically in a multiverse theory, one realizes that there are major loopholes in the naturalness argument. The main loophole is that it completely ignores the measure factor. Considering each of the three factors in Equation (1), it is reasonable to expect $A(i)$ to be independent of the supersymmetry breaking scale as long as this is well above the scales (atomic, nuclear, etc.) which enter anthropic arguments. As for the cosmological factor $U(i)$, it is true that some of the ingredients in the eternal inflation discussion of Section 3 depend on the supersymmetry breaking scale, but not in a direct way and as yet there is no clear argument either way about such dependence.

However, let's grant for the sake of argument that $U(i)$ is not correlated with the supersymmetry breaking scale. Even so, if we cannot estimate the vacuum counting measure, we still cannot say whether string theory predicts low energy supersymmetry. To see this, suppose there are two classes of vacua, class A with a mechanism such as supersymmetry which leads to low Higgs mass in a large fraction of vacua, and class B in which this can only come from fine-tuning, so that one expects a fraction 
$10^{-34}$ of class B vacua to realize a low Higgs mass. Nevertheless. if the counting measure Equation (4) favored class B by a factor greater than $10^{34}$, then string theory would predict we live in a class B vacuum. Thus, one can envisage a scenario in which the discovery of low energy supersymmetry would be evidence against string theory! This was realized around $2004[19,20]$ and efforts were then made to estimate the counting part of this measure, reviewed in [5]. Although this discussion was not conclusive, my own opinion at present [21] is that the suggestions made at that time for a large number of vacua with high scale supersymmetry breaking were not confirmed, and that vacuum counting will prefer low energy supersymmetry, though this is by no means proven.

In any case, the scenario in which low energy supersymmetry provides a natural solution to the hierarchy problem meets another fatal difficulty in string theory, the moduli problem [22,23]. This starts with the observation that scalars with gravitational strength couplings to the Standard Model with mass $M \lesssim 30 \mathrm{TeV}$ lead to an overabundance of dark matter which is incompatible with the theory of big bang nucleosynthesis. Then, one can show on very general grounds $[13,24]$ that string compactifications always contain at least one scalar with $M \lesssim M_{\text {susy }}$, placing a lower bound $M_{\text {susy }} \gtrsim 30 \mathrm{TeV}$. While solutions to the moduli problem have been suggested, they are unnatural in other ways. Since taking $M_{\text {susy }} \sim 30 \mathrm{TeV}$ brings the fine-tuning required to reproduce the observed Higgs mass down to a modest $10^{-3}$ or so, this relatively straightforward scenario seems plausible. Thus, if we accept all this, a combination of phenomenological and landscape arguments favor low energy supersymmetry around the scale $30-100 \mathrm{TeV}$. If so, while supersymmetry may be out of reach at LHC, a next generation accelerator could discover it.

After the supersymmetry breaking scale, arguably the next most important "axis" along which string vacua differ in a potentially testable way is the number of hidden sectors, meaning matter sectors whose couplings to the Standard Model are comparable to that of gravity. This could be because the couplings are suppressed by powers of large energy scales $M_{\text {Planck }}$ or $M_{G U T}$, or it could be because the couplings violate an approximate symmetry. An example of the former might be an additional $x$ sector, perhaps similar to the Standard Model. An important example of the latter is the axion, a scalar field whose interactions respect a shift symmetry $\phi \rightarrow \phi+a$ to all orders in perturbation theory, but which is violated nonperturbatively. Such axions are generic in string theory [25].

An attractive feature of the heterotic models of [1] was that, not only did they naturally lead to grand unified theories, they also led to a simple hidden sector (the extra $E_{8}$ ). However, this simplicity did not survive the second superstring revolution, and the models which solve the cosmological constant problem have many hidden sectors as well as hundreds or more axions. These are all dark matter candidates, and can lead to many other testable predictions. A very striking example is the superradiance of rotating black holes discussed in [26], which can rule out axions in certain mass windows without any other assumptions. In fact, it seems likely that generic string vacua with hundreds of axions whose masses are uniformly distributed on a log scale will be ruled out this way.

This axis of variation can be referred to as the "simplicity-complexity" axis. Is the typical string vacuum simple, having few matter fields, or complex, having many? One would expect on general grounds that there will be many more complex configurations than simple ones because they have more parameters to vary, so that the complex vacua will dominate the vacuum counting measure. Indeed, this is what has come out so far from the arguments we will discuss in Section 2.

Even if the vacuum counting measure leads to definite predictions, we still need to estimate the cosmological factor $U(i)$, for which we need to study the quantum cosmology of string/M theory. A satisfactory discussion of this would start from a precise definition of string/M theory which includes both the quasi-realistic vacua and the other regimes that are important in quantum cosmology, such as eternally inflating solutions and possible non-geometric solutions. Since at present we have no such definition, this discussion is also in its infancy. What has mostly been done is to make contact with the analysis based on eternal inflation and vacuum tunneling, developed in many works of which $[27,28]$ have particularly influenced the string theory work. It seems clear to most researchers that string/M theory satisfies the general conditions required to realize eternal inflation, and thus, by the arguments 
of that literature, one expects the measure to be dominated by that part of the multiverse, which is described by eternal inflation. Granting this, one can try to use the master equation developed in that work to determine the measure factor. This was studied in the works [28,29], with general results we outline in Section 3.

\section{String Compactification and the Effective Potential}

There are many good reviews of string compactification such as [30], and the general picture of the subject has been stable for about a decade now, so we refer readers who want the basics to the reviews and here give a very brief overview with comments on the current situation.

Most work on compactification of string/M theory follows the general approach initiated in the 1970s for higher dimensional supergravity theories. One starts with the low energy limiting effective field theory, a ten- or eleven-dimensional supergravity, and adds various terms to the action which describe important stringy and quantum effects. One then assumes that space-time takes the form $M_{4} \times K$, where $M_{4}$ is a maximally symmetric four-dimensional space-time and $K$ is a compact six- or seven-dimensional manifold, and looks for solutions of the quantum corrected equations of motion.

To get started on this, almost always one takes a manifold $K$ with a Killing spinor, in other words which preserves some supersymmetry in $M_{4}$. This would seem to be a very restrictive assumption which rules out de Sitter $M_{4}$ from the start. Since the simplest way to model the observed dark energy is as a positive c.c., and we also want to find solutions which describe inflation, it is important to construct de Sitter vacua; indeed, when we talk about quasi-realistic vacua in this survey, we will include the condition of a small positive cosmological constant. Thus, this starting point should be examined critically.

Could there be other solutions not based on supersymmetry and Killing spinors? A heuristic argument for supersymmetry at and near the compactification scale is that instabilities are very generic - this is one of the main lessons of the literature on string compactification. One wants mechanisms to stabilize the solution, and supersymmetry tends to do this because the potential is a sum of squares (we will talk about the negative term in supergravity below). Nevertheless, constructions not relying on special holonomy and Killing spinors have been suggested-see particularly [31] and references there. How can we evaluate them?

Mathematically, one expects $K$ to fall into a "trichotomy" of positive, zero or negative Ricci curvature [32]. For nonzero curvature, one needs internal stress-energy, and for negative curvature this has to violate positive energy conditions, leading to a restrictive no-go theorem [33]. Of course, there are many positive curvature solutions-these naturally have negative c.c. proportional to the curvature of $K$, and furthermore the diameter of $K$ is related to its curvature as $R \sim c / d^{2}$ for some order one $c$, so realizing small negative c.c. generally makes the diameter large, eventually running into the phenomenological bounds from non-observation of "Kaluza-Klein particles" or even from the validity of the inverse square law of gravity down to the few micron scales. Considerations along these lines also lead to arguments that only a finite number of vacua satisfy the diameter constraint [11]. This leaves the case of Ricci flat $K$, which is accessible to mathematical analysis. All known Ricci flat manifolds in fact have special holonomy and Killing spinors, and proving this for four-dimensional $K$ is an active research topic. If this is always so, then there is a strong argument for supersymmetry at some energy scale. Then, although the scale might be high, one can estimate the dependence of the vacuum counting measure on this scale, and make a clear argument for whether low or high scale is favored.

Once we assume supersymmetry, there is a short list of constructions. Probably, the most general is $\mathrm{F}$ theory compactified on a Calabi-Yau fourfold, and the others are $\mathrm{M}$ theory compactified on a manifold of $G_{2}$ holonomy, and the five superstrings compactified on Calabi-Yau threefolds. By "most general", we mean the construction which realizes the most choices and makes the physics clearest from a purely geometric analysis, based on supergravity plus corrections. The conjecture is that, once we take all stringy and quantum effects into account, all the constructions lead to the same landscape, 
but the simplicity of a geometric analysis is a great advantage. Having said this, it should also be said that working with $\mathrm{F}$ theory requires going beyond perturbative string theory and many physical computations are still only possible in the superstring $/ \mathrm{CY}_{3}$ constructions.

In the Calabi-Yau constructions, one is relying on the famous theorem of Yau which guarantees a Ricci-flat metric, which is uniquely determined given a finite number of parameters or "moduli" which include the overall volume and the complete list of integrals of two preferred differential forms (the Kähler form and the holomorphic three-form or four-form) over respective bases of homology classes. One then must make many additional discrete and continuous choices-higher dimensional branes can fill $M_{4}$ and wrap cycles of $K$; these can carry holomorphic vector bundles; one can postulate generalized magnetic fields or "flux." While all of these choices are classified topologically, the observed fact that they are finite in number-indeed, one can find Calabi-Yau compactifications with arguably "the largest gauge group" [34] or "the largest number of flux vacua" [4] — does not follow from topology but rather from still somewhat mysterious algebraic geometric arguments.

At first sight, the most striking thing about these compactifications is how many fields they have compared to the Standard Model. While the particle physicists of the 1930s were surprised by the muon, asking "Who ordered that?", string theorists should be even more surprised by the thousands or even hundreds of thousands of fields in the most complex compactifications. Still, this is not in contradiction with observation as these fields will be light only in the most symmetric compactifications; symmetry breaking can give large masses to almost all of these fields. Others could be in hidden sectors, coupling only gravitationally to the Standard Model and to the inflaton, so that they are not produced in reheating. While there are also compactifications with relatively few fields, certainly we should look for evidence of many fields in our universe. One interesting possibility is that compactifications often contain many axions, supersymmetry partners of the moduli, which only get masses nonperturbatively and could realize a log uniform mass spectrum. This leads to many potential signatures, as first discussed at length in [26].

Another approach to making predictions is to argue that no string theory compactification, or perhaps even no quantum theory of gravity, can have a particular low energy property. Some of the first examples were the argument that global symmetries must be broken in quantum gravity [35], and the weak gravity conjecture [36], which requires the existence of light charged particles. A recent survey of these "swampland" arguments is [37].

To more deeply understand the physics of a string compactification, one needs to derive the low energy effective theory. One can push this very far using powerful methods of algebraic geometry, especially the theory of variation of Hodge structure which allows for computing the periods of the holomorphic three and four-form as a function of the moduli. This determines the flux superpotential [38], which is a major part of the complete effective superpotential, and which by string dualities encodes a great deal of nonperturbative four-dimensional physics, in particular the nonperturbative effects which were called upon in work on dynamical supersymmetry breaking in beyond the Standard Model physics.

A central property of the string landscape, as discovered by Bousso and Polchinski [12], is that it contains the large set of vacua with different values of the vacuum energy required by the anthropic solution to the cosmological constant problem. They showed this by looking at vacua with nonzero values of higher form gauge fields, constant on $M_{4}$ and consistent with its maximal symmetry, but contributing to the vacuum energy by the usual Maxwell-type magnetic terms, in Ilb compactification schematically

$$
V=\int_{K} d^{6} y \sqrt{g} F \wedge * F+e^{-2 \phi} H \wedge * H
$$

where $F$ and $H$ are three-forms. This expression depends on the metric on $K$, the "dilaton" scalar field $\phi$, and many other fields which we have suppressed. Now, it is not possible to explicitly solve the 
equations for these fields and directly evaluate $V$. However, what matters for their argument is that configuration space breaks up into sectors with fixed values of the quantized "fluxes"

$$
N_{R R}^{i} \equiv \int_{\Sigma_{i}} F ; \quad N_{N S}^{i}=\int_{\Sigma_{i}} H,
$$

and that within each sector there is a solution. In this case, one can model the dependence of the vacuum energy on the flux quanta $N$ as a quadratic function

$$
V \sim M^{4} \sum_{i, j} G_{i, j} N^{i} N^{j}-\Lambda_{0}
$$

where $M$ is an energy scale set by the quantization condition Equation (6), and $G_{i, j}$ is determined by the weak dependence of the other fields on the $N^{\prime}$ s. Neglecting this and assuming that the $G_{i, j}$ take generic fixed values, one finds a "discretuum" of possible values of $V$ which will be uniformly distributed near $V \sim 0$ with average spacings $M^{4} / \bar{N}^{2 b_{3}}$. Here, $b_{3}=\operatorname{dim} H^{3}(K ; \mathbb{Z})$ is the third Betti number of $K$, which ranges from 4 up to 984 . The maximal flux $\bar{N}$ is set by string theoretic details (the "tadpole condition") but typically ranges from 10 to 100 , so, even with $M \sim M_{\text {Planck }}$ (it is smaller), this construction can easily achieve the required spacing.

While this is a good qualitative argument, to go any further and especially to say anything about the distribution of supersymmetry breaking scales, one needs to put in more about string theory. Thus, let us write out the flux superpotential for IIb string compactified on $\mathrm{CY}_{3}$; it is

$$
W=\sum_{1 \leq i \leq b_{3}}\left(N_{R R}^{i}+\tau N_{N S}^{i}\right) \Pi_{i}(\vec{z})
$$

The flux numbers $N_{R R}^{i}, N_{N S}^{i}$ are integers, $\tau$ and $\vec{z}$ are the moduli, and the $\Pi_{i}$ are the periods of the holomorphic three-form, which can be written explicitly as generalized hypergeometric functions, using methods of mirror symmetry [39]. Following the rules of $4 \mathrm{~d}, N=1$ supergravity, this determines the effective potential, as a sum of terms quadratic in $W$. This can be shown to be equal to the minimum of the potential terms for higher form gauge fields $F$ and $H$, schematically with $F$ and $H$ satisfying the Dirac quantization conditions at the solutions of the equations of motion. Thus, we can compute the exact effective potential due to flux, despite our inability to explicitly solve for the Ricci flat metric or the other fields on $K$.

Besides its computability, the main point we want to make about the flux superpotential here is that it provides a large number of independent contributions to the vacuum energy that preserve supersymmetry. This has the important consequence that, as explained in [5], the distribution of cosmological constants is independent of the supersymmetry breaking scale. One might have thought a priori that this distribution was uniform only for $|\Lambda|<M_{\text {susy }}^{4}$, in which case the need to get small cosmological constant would favor low energy supersymmetry. Because $\Lambda$ also gets supersymmetry preserving contributions, this is not the case.

By itself, the physics of fluxes stabilizes many but not all of the moduli. In particular, one finds that the overall volume of $K$ is not fixed. While it must eventually be fixed when supersymmetry is broken, having this happen at observable scales is very problematic and it is much easier to get a metastable vacuum with reasonable cosmology if all of the moduli are stabilized before supersymmetry breaking. Such models have been constructed, but they rely on partially understood stringy and quantum corrections to the effective potential. Thus, the further discussion is not yet based on exact results but on expansions around controlled limits and physical intuition. 
There are two popular classes of moduli stabilizing potentials, the KKLT ${ }^{3}$ construction [40] and the large volume construction [41,42]. Both are most often applied to type IIb and F theory compactifications. In KKLT, one relies on nonperturbative effects in the superpotential. These include the very well understood nonperturbative superpotentials of supersymmetric four-dimensional gauge theory, as well as stringy instanton effects (in some cases, these are two dual descriptions of the same thing). These depend on the Kähler moduli and thus a fairly generic superpotential will have supersymmetric minima at which these are stabilized (which are necessarily anti de Sitter (AdS)). Because the new terms are nonperturbative, they can be very small even if the compactification manifold is string scale, and these constructions can make contact with the original picture of dynamical supersymmetry breaking as envisioned in [1]. The analysis also benefits from the protected nature of the superpotential, which makes computations tractable and holds out the possibility of exact results.

In the large volume construction, one relies on a particular correction to the Kähler potential, which appears in world-sheet perturbation theory [43], to find an effective potential with a nonsupersymmetric AdS minimum. While this may sound a bit arbitrary, this correction is believed to be protected as well. Then, because the terms which are balanced to find a minimum are perturbative, one finds a universal structure of the potential near the minimum, and energy scales which are simple powers of the volume of the internal dimensions. This leads to rather different predictions from the KKLT and dynamical supersymmetry breaking models.

Once one has stabilized the moduli, the next step in the analysis is to argue that the vacuum exhibits supersymmetry breaking and has a positive cosmological constant (we refer to these as de Sitter or dS vacua). From a conceptual point of view, once one has accepted that the main point is to show that the effective potential has these properties, such vacua are expected to be generic as there are many candidate supersymmetry breaking effects which make positive contributions to the vacuum energy, while there is nothing special about the value zero for the sum of all of these effects. However, what is special about the value zero is that the controlled limits of the problem (large volume and classical limits) about which we must expand around to do our computations have zero vacuum energy. Furthermore, these limits can be reached by varying moduli (the overall volume modulus and the dilaton). Thus, one needs not only positive energy for the candidate vacuum, but an even larger energy for all the nearby configurations, so that it will be a local minimum. Even if we have this, there will inevitably be an instability to tunneling back to large volume. One of the main points of the original KKLT work was to argue that this decay is usually highly suppressed.

The original KKLT work also argued that one could start with a supersymmetric AdS compactification, which is an easier theoretical problem, and then obtain a dS compactification by inserting an anti D3-brane into the compactification. Since one has combined branes and antibranes which can annihilate, this will always break supersymmetry, while one can show that this annihilation process is also very slow [44]. This mechanism was also called upon in [42].

The anti D3-brane has the advantage of simplicity, but any additional source of positive energy, such as one would get from sufficiently many distinct fluxes or nonperturbative effects, could play the same role. Indeed, in [13], it was argued that generic flux potentials depending on a single modulus would have metastable supersymmetry breaking critical points. If such were the case in models with large numbers of moduli, one might find a vast number of high scale supersymmetry breaking vacua. This point was considered in [45] who introduced a toy random matrix model of the flux superpotential, and showed in their model that such critical points are almost always unstable unless almost all the moduli have masses well above the supersymmetry breaking scale. This is good news for hopes of observing low energy supersymmetry; however, the assumptions behind the toy model are not beyond criticism [46,47]. Still, since one can get metastable minima even with a potential depending on a single field, and there is no way for local physics to distinguish between small positive and negative

3 Kachru, Kallosh, Linde and Trivedi. 
values of the c.c. (this was one of the central points of [6,7]), it would require a vast and mysterious conspiracy to eliminate all metastable de Sitter compactifications.

\section{Problems with the String Compactification Analysis}

At present, these analyses go beyond the controlled computations and not all string theorists are convinced, see [48-52] for some of the criticisms and [53-55] for a few of the many rebuttals. I cannot do justice to this controversy here but will only comment that to my mind; the criticisms fall into three classes. One class is to my mind superficial and is largely based on properties of the large volume limit and the difficulty of the extrapolation we must do to get to the regime where metastable de Sitter vacua can exist, which as was known for a long time must involve significant stringy and quantum corrections [56-59]. Arguments such as that of [51] which postulate simple constraints on all possible vacua without a mechanism which enforces them on all vacua are not persuasive.

Another class of criticisms [60-62] tries to construct explicit solutions with quantum corrections and meets specific difficulties. For example, after inserting an antibrane to uplift to positive vacuum energy, one must check that the backreaction of the metric and other fields does not make the total vacuum energy negative. While technical, it is crucial to settle such points as these explicit constructions are the foundation for the entire discussion. It is very possible that, on properly understanding them, the statistics of vacua will look rather different. For example, it might turn out that complex vacua (on the simplicity-complexity axis of Section 1) are much harder to stabilize than simple ones, disfavoring them. ${ }^{4}$

The third class of criticism [48] is deep and starts from the postulate that the holographic dual, whose nature depends crucially on the large scale structure of space-time and thus on the sign of the cosmological constant, is more fundamental than the approximate descriptions of string compactification which we work with now. In this view, because the duals of AdS and dS vacua are so different, trying to relate the two types of construction is misguided. This idea, which contradicts very basic tenets of nongravitational physics, can only be properly judged when we have a more complete understanding of string/M theory.

In my opinion, a convincing construction of metastable supersymmetry breaking de Sitter vacua and a good understanding of their physics must be founded on a controlled computation of the effective potential. Although major advances will be required, there is no known obstacle to someday computing the exact superpotential for a wide range of supersymmetric string compactifcations. The same cannot be said (yet) for the Kähler potential, the other necessary ingredient to determine the effective potential (the final ingredient, the $\mathrm{D}$ terms, are determined by the symmetries of the Kähler potential). Most work, both in string compactification and in the study of four-dimensional gauge theory, uses perturbative approximations to the Kähler potential. This often can be justified by other physical arguments, but it is a weak point. For some gauge theories in the large $N$ limit, one can get the effective potential using gauge-gravity duality. This at least points the way to other techniques, and I believe there will be progress on controlled calculations of the Kähler potential and thus the full effective potential, though perhaps not soon.

\section{Eternal Inflation and Measure Factor}

We will assume that the reader is familiar with the theory of eternal inflation, if not he or she is directed to one of the many reviews such as $[64,65]$. Although there is controversy about the predictions of this theory, I generally follow the analysis given in [27] and Section 2 of [66] has a brief review from this point of view. To summarize the results of this theory, if we assume that cosmological dynamics is dominated by eternal inflation and semiclassical tunneling between metastable vacua with positive c.c.,

4 There are several arguments that complex vacua might be disfavored; see [63]. 
we can show that the stochastic evolution of the multiverse is governed by a linear master equation for $N_{i}(t)$, the number of pocket universes of type $i$ at time $t$. This equation takes the form

$$
\frac{d}{d t} N_{i}(t)=\gamma_{i} N_{i}(t)+\sum_{j} \Gamma_{j \rightarrow i} N_{j}(t)-\sum_{j} \Gamma_{i \rightarrow j} N_{i}(t)
$$

where $\gamma_{i}$ represents the exponential growth due to inflation, and $\Gamma_{i \rightarrow j}$ is the tunneling rate from universe type $i$ to type $j$. Usually, one uses the Coleman-DeLuccia (CDL) formula for these rates [67], calculated by describing the tunneling in terms of a domain wall solution and applying semiclassical quantum gravity. We will not quote this formula except to comment that the rates are typically exponentially small (as usual for tunneling rates) and even doubly exponentially small (as we explain below).

The right-hand side of Equation (9) can be rewritten as a matrix acting on $N_{i}(t)$, so that the equation can be solved by diagonalizing this matrix. In the large $t$ limit, except for a set of initial conditions of measure zero, the solution will approach the dominant eigenvector; in other words, the eigenvector of the matrix with the largest eigenvalue. The $i$ 'th component of this eigenvector is then the factor $U(i)$ in Equation (1). Note, however, that the time required to reach this limit can be extremely long, a point we will return to below.

The structure of this dominant eigenvector depends very much on the tunneling rates $\Gamma$. We can illustrate this by considering two extreme cases. In the first, each rate $\Gamma_{i \rightarrow j}$ is either zero (tunneling is forbidden) or a constant rate $\epsilon$. In this case, the landscape is completely described by an undirected graph (the rates are expected to be symmetric), and techniques of graph/network theory can be useful. A simplified landscape of this type of F theory vacua was studied in [29], and it was found that the high dimensionality of the landscape led to exponentially large ratios between the limiting $U(i)$ 's.

The other extreme case is when one of the metastable vacua is far longer lived than the others, as then the dominant eigenvector will be highly concentrated on this vacuum. This leads to the following prescription for the measure factor: one must find the longest-lived metastable vacuum, call this $V_{0}$. Then (up to subleading factors depending on $\gamma_{i}$ and an irrelevant overall factor), we have

$$
U(i) \sim \Gamma_{0 \rightarrow i} \quad \forall i \neq 0 .
$$

Now, it is very plausible that $V_{0}$ is not anthropically acceptable $(A(0)=0)$, so the upshot is that the favored vacua are "close to $V_{0}$ " in the sense that they can be easily reached from it by tunneling.

An analysis of the cosmology of a Bousso-Polchinski landscape along these lines was made in [28]. They argued that the hypothesis of a single longest-lived metastable vacuum will be generic under the following assumptions. First, we only consider vacua with $\Lambda>0$; all tunnelings to vacua with $\Lambda \leq 0$ are ignored as they do not lead to inflating bubbles. Second, we use the Coleman-DeLuccia tunneling rates for $\Gamma_{i \rightarrow j}$. These include a factor of exp - $\left(M_{\text {Planck }}^{4} / \Lambda_{i}-M_{\text {Planck }}^{4} / \Lambda_{j}\right)$, so "uptunnelings" with $\Lambda_{j}>\Lambda_{i} \approx 0$ are highly suppressed. Indeed, a realistic landscape must contain vacua with $\Lambda_{i} \sim 10^{-120} M_{\text {Planck }}^{4}$ and, in this sense, these tunnelings are doubly exponentially suppressed.

How does one find the longest-lived metastable vacuum? From the above, it might sound like it is the vacuum with the smallest positive $\Lambda$, which would be bad news as finding this one is computationally intractable [68]. Fortunately, there are other equally important factors in the tunneling rate. In fact, one can make suggestive arguments that the longest lived metastable vacuum is the one with the smallest scale of supersymmetry breaking [69], and that this comes from the compactification with the most complex topology [21]. If so, this would narrow down the search tremendously and might well lead to testable predictions-for example, that we should expect many hidden sectors associated with the complex topology of the extra dimensions.

Actually deriving Equation (9) from semiclassical gravity and making these arguments precise requires defining a time coordinate $t$ and understanding the long time limit, which is quite ambiguous and this leads to paradoxes. By now, many definitions of the time coordinate as well as variations on 
the argument have been studied (a review is in [65]). Although the topic is by no means settled, many of the definitions and alternate arguments (for example, that of [70]) do seem to lead to the claim that the term $U(i)$ in the measure factor is as we just discussed, the tunneling rate from the longest-lived metastable vacuum.

\section{Problems with the Eternal Inflation Analysis}

Let us summarize the claim of the previous subsection. Starting with very general assumptions, namely that the dynamics of the multiverse is dominated by the process of eternal inflation, and that this process runs long enough to reach an equilibrium, one can derive a well-defined cosmological measure factor $U(i)$ expressed in terms of quantities we can actually hope to compute, namely the set of metastable dS vacua, their cosmological constants and the tunneling rates between them. This would be very impressive were it not for some crucial gaps in the argument. These gaps are known and discussed in the literature, and we suggest [70] as a good reference.

A very serious gap is that the analysis leading to Equation (9) was all done in four-dimensional semiclassical gravity. While it should also hold for the string/M theory solutions we are discussing, with four large dimensions and the other spatial dimensions stabilized to a fixed small manifold, of course, this is a tiny part of the full configuration space, in which any subset of the dimensions could be large. The only excuse I know of for ignoring this major omission is that it is plausible that having four large dimensions is the only way to get eternal inflation. The physics called upon in the dS vacuum constructions we cited does not generalize to higher dimensions, while general relativity behaves very differently in fewer dimensions. Of course, this argument is not entirely convincing. Even if we grant it, it might be that the dominant tunneling processes between the four-dimensional vacua pass through the larger configuration space of higher dimensional or even non-geometric configurations.

Another important gap is in the treatment of AdS vacua. Now, in Section 2, we stressed that, from the point of view of local physics, there can be no essential difference between dS, AdS and Minkowski vacua; indeed, the uncertainty principle makes it impossible to distinguish between them without making measurements at lengths $L \gg|\Lambda|^{-1 / 4}$. On the other hand, the global structures of these different space-times are very different, and they enter into the arguments for Equation (9) very differently. We know there are many supersymmetric AdS and Minkowski vacua of string theory, as we discussed in Section 2. According to CDL and other general arguments, supersymmetric vacua are absolutely stable to tunneling, so their decay rates are $\Gamma=0$ (they are "terminal vacua"). Granting this, it is straightforward to take them into account, and the claim for the measure factor remains true. However, while solutions describing tunneling from dS to Minkowski vacua are non-singular and this treatment seems sensible, the solutions describing tunneling from dS to AdS vacua are singular, ending in a "big crunch" [67]. While these transitions are usually left out, it could be that AdS vacua do somehow tunnel back to dS and must be taken into account to properly define Equation (9).

Another potential problem with the whole picture arises from the double exponentially suppressed nature of tunneling rates. It is that the ratios between probabilities $P(i)$ in Equation (1) can be so large that vacua with very small values of $A(i)$ become important. This was pointed out in [28] which studied Equation (9) in an explicit Bousso-Polchinski flux landscape (Equation (7)). They found that, because of the wide variation of tunneling rates, the factor $U(i)$ was very irregular, in a way one can model as a roughly uniform constant plus a much larger fluctuating random variable. Now, because in inflationary cosmology observables such as $\delta \rho / \rho$ have quantum fluctuations, a vacuum with $\Lambda$ violating the anthropic bound of [6] does not have $A=0$. Rather, there is an exponentially small probability for a density enhancement to allow structure formation in a small part of the universe. If this suppression were overwhelmed by a double exponential, it could turn out that most observers live in small overdense regions of an otherwise empty universe. Even worse, to decide whether this is the case and make any predictions at all, one must compute the $A(i)$ to the same accuracy as the $U(i)$. Given the difficulty of understanding $A(i)$, this is problematic. 
Universes with very small but nonzero $A(i)$ were referred to as "marginally hospitable" in [66] and lead to problems for mulitverse cosmology. The most extreme is the famous "Boltzmann brain" paradox [71,72], which points out that there is a nonzero (though doubly exponentially small) probability to create a local region of space-time containing any specified matter distribution-say, a disembodied brain imagining our universe-as a random thermodynamic fluctuation. Granting the advances in biology and artificial intelligence since Boltzmann's time, we can imagine more likely ways that this could happen, through fluctuations which create a local region in which evolution and/or engineering produces observers. Although very far-fetched from any point of view, if the space-time volume of the multiverse is doubly exponentially large, these could be the most numerous observers.

The main point is that double exponential probabilities are very small and even the most unlikely-seeming scenarios could be more likely. I would go so far as to advocate the principle that any theory which is complex enough to describe universal computing devices and whose predictions depend on differences between probabilities less than $10^{-10^{10}}$ and zero cannot be believed because the analysis needed to rule out other comparably rare events is computationally intractable (and perhaps even undecidable). However, I do not know how to make this argument precise.

What is clear is that the relevance of these double exponentially small rates to quantum cosmology depends on the assumption that the dynamics of multiverse cosmology runs to equilibrium. This is another way of stating the assumption of the large time limit made in the paragraph following Equation (9). The time taken to reach equilibrium can be estimated as the spectral gap of the matrix in Equation (9), which is more or less the second longest lifetime of a metastable vacuum. This will also be a double exponential, at least $10^{10^{120}}$ Planck times and probably much longer. This is the context in which I invite the reader to reflect on the above doubts about double exponentials.

This observation brings us to what I consider to be the main philosophical objection to the multiverse. ${ }^{5}$ It is that, in postulating a multiverse, we are postulating a far larger and more complex structure than any model of what we can actually observe. This does not make the multiverse idea wrong, but it does motivate looking for a formulation of a multiverse theory without this property, or at least one that minimizes the complexity of the multiverse. Clearly, a good first step in this direction would be to have some way to quantify the complexity of the multiverse.

This question was first raised in [66], which also proposed a way to answer it. The conceit suggested there is to imagine that the multiverse is being simulated by a powerful quantum supercomputer, which is searching for vacua that satisfy the conditions required for the emergence of structure and observers, or at least those conditions which can be tested within a fixed amount of time (we refer to these as "hospitable" vacua). ${ }^{6}$ Following this idea leads to a definition of the complexity of the multiverse in terms of the computational resources (number of quantum gate operations) required to do the simulation, analyzing the intermediate results and controlling the search. At least in semiclassical quantum gravity, one can use the freedom to choose a time coordinate to express the choice of which parts of the multiverse to simulate. Given an algorithm for making this choice, the corresponding measure factor $U(i)$ is simply the probability with which the vacuum $V_{i}$ will be the first hospitable vacuum found by the supercomputer. One can even argue that there is a preferred time coordinate, "action time," which (following a conjecture in [73]) counts the number of gate operations required for a simulation. According to this conjecture, and consistent with the earlier analysis of [74], the observable universe could be simulated with $\sim 10^{120}$ operations. An "economical" multiverse proposal would be one which does not require too many more operations than this.

5 See also the article [8] in this issue.

6 Our point was not to claim that the multiverse is a simulation, in fact we explicitly postulate that no local observer can tell that the multiverse is being simulated. Rather, in the spirit of computational complexity theory, the point is to define the complexity of the multiverse in terms of a "reduction" to another problem whose complexity we know how to define, namely that of running a specified quantum computer program. 
On thinking about this, it is clear that the standard eternal inflation treatment based on Equation (9), with its double exponential time to reach equilibrium, is very far from an economical multiverse. One can easily improve its efficiency by making simple changes to the search algorithm. For example, after simulating a given vacuum for $10^{100}$ years, if no interesting tunneling or other events have been detected, the computer simply abandons simulating that branch of the multiverse and switches to another. This particular variation can be analyzed as a modification of Equation (9) and one finds drastically different dynamics, which arguably lead to a measure factor concentrated on the vacua which are close to (in the sense of tunneling rates) whatever preferred initial conditions string/M theory might provide for the multiverse. If the initial conditions are simple, this would favor the "simple" side of the simplicity-complexity axis of Section 1. Further analysis of the resulting predictions will have to wait until we know something about these initial conditions, which may require finding a new formulation of string/M theory. However, this is to be expected of any approach which abandons the assumption that the dynamics of the multiverse is at equilibrium.

One sees that our understanding of the string landscape is still in its early exploratory days. Let me conclude by repeating what I think is the most important question about the multiverse:

Is the multiverse in equilibrium or not?

Funding: This research received no external funding.

Acknowledgments: I thank many people for discussions on these topics, particularly Bobby Acharya, Nima Arkani-Hamed, Sujay Ashok, Tom Banks, Raphael Bousso, Frederik Denef, Michael Dine, Bogdan Florea, Antonella Grassi, Brian Greene, Alan Guth, Shamit Kachru, Renata Kallosh, Gordy Kane, Andrei Linde, Bernie Shiffman, Eva Silverstein, Wati Taylor, Cumrun Vafa, Alex Vilenkin, Steve Zelditch and Claire Zukowski.

Conflicts of Interest: The author declares no conflict of interest.

\section{References}

1. Candelas, P.; Horowitz, G.T.; Strominger, A.; Witten, E. Vacuum Configurations for Superstrings. Nucl. Phys. B 1985, 258, 46-74. [CrossRef]

2. Witten, E. String theory dynamics in various dimensions. Nucl. Phys. B 1995, 443, 85-126. [CrossRef]

3. Denef, F.; Douglas, M.R. Distributions of flux vacua. J. High Energy Phys. 2004, 2004, 72. [CrossRef]

4. Taylor, W.; Wang, Y.N. The F-theory geometry with most flux vacua. J. High Energy Phys. 2015, $2015,164$. [CrossRef]

5. Douglas, M.R.; Kachru, S. Flux compactification. Rev. Mod. Phys. 2007, 79, 733. [CrossRef]

6. Weinberg, S. The Cosmological Constant Problem. Rev. Mod. Phys. 1989, 61, 1. [CrossRef]

7. Polchinski, J. The Cosmological Constant and the String Landscape. arXiv 2006, arXiv:hep-th/0603249.

8. Heller, M. Multiverse-Too Much or Not Enough? Universe 2019, 5, 113. [CrossRef]

9. Sandora, M. Multiverse Predictions for Habitability: The Number of Stars and Their Properties. Universe 2019, 5, 149. [CrossRef]

10. Douglas, M.R. The Statistics of string / M theory vacua. J. High Energy Phys. 2003, 2003, 46. [CrossRef]

11. Acharya, B.S.; Douglas, M.R. A Finite landscape? arXiv 2006, arXiv:hep-th/0606212.

12. Bousso, R.; Polchinski, J. Quantization of four form fluxes and dynamical neutralization of the cosmological constant. J. High Energy Phys. 2000, 2000, 006. [CrossRef]

13. Denef, F.; Douglas, M.R. Distributions of nonsupersymmetric flux vacua. J. High Energy Phys. 2005, $2005,61$. [CrossRef]

14. Acharya, B.S.; Bobkov, K.; Kane, G.L.; Shao, J.; Kumar, P. The G(2)-MSSM: An M Theory motivated model of Particle Physics. Phys. Rev. D 2008, 78, 065038. [CrossRef]

15. Marsano, J.; Saulina, N.; Schafer-Nameki, S. Gauge Mediation in F-Theory GUT Models. Phys. Rev. D 2009, 80, 046006. [CrossRef]

16. Dundee, B.; Raby, S.; Westphal, A. Moduli stabilization and SUSY breaking in heterotic orbifold string models. Phys. Rev. D 2010, 82, 126002. [CrossRef]

17. Aparicio, L.; Cerdeno, D.G.; Ibanez, L.E. Modulus-dominated SUSY-breaking soft terms in F-theory and their test at LHC. J. High Energy Phys. 2008, 2008, 99. [CrossRef] 
18. Ibanez, L.E. From Strings to the LHC: Les Houches Lectures on String Phenomenology. arXiv 2012, arXiv:1204.5296.

19. Douglas, M.R. Statistical analysis of the supersymmetry breaking scale. arXiv 2004, arXiv:hep-th/0405279.

20. Susskind, L. Supersymmetry breaking in the anthropic landscape. In From Fields to Strings: Circumnavigating Theoretical Physics; World Scientific: Singapore, 2005; Volume 3, pp. 1745-1749.

21. Douglas, M.R. The string landscape and low-energy supersymmetry. Les Houches Lect. Notes 2015, 97, 315.

22. Banks, T.; Kaplan, D.B.; Nelson, A.E. Cosmological implications of dynamical supersymmetry breaking. Phys. Rev. D 1994, 49, 779. [CrossRef] [PubMed]

23. de Carlos, B.; Casas, J.A.; Quevedo, F.; Roulet, E. Model independent properties and cosmological implications of the dilaton and moduli sectors of 4-d strings. Phys. Lett. B 1993, 318, 447-456. [CrossRef]

24. Acharya, B.S.; Kane, G.; Kuflik, E. String Theories with Moduli Stabilization Imply Non-Thermal Cosmological History, and Particular Dark Matter. arXiv 2010, arXiv:1006.3272.

25. Svrcek, P.; Witten, E. Axions In String Theory. J. High Energy Phys. 2006, 2006, 51. [CrossRef]

26. Arvanitaki, A.; Dimopoulos, S.; Dubovsky, S.; Kaloper, N.; March-Russell, J. String Axiverse. Phys. Rev. D 2010, 81, 123530. [CrossRef]

27. Garriga, J.; Schwartz-Perlov, D.; Vilenkin, A.; Winitzki, S. Probabilities in the inflationary multiverse. J. Cosmol. Astropart. Phys. 2006, 2006, 17. [CrossRef]

28. Schwartz-Perlov, D.; Vilenkin, A. Probabilities in the Bousso-Polchinski multiverse. J. Cosmol. Astropart. Phys. 2006, 606, 10. [CrossRef]

29. Carifio, J.; Cunningham, W.J.; Halverson, J.; Krioukov, D.; Long, C.; Nelson, B.D. Vacuum Selection from Cosmology on Networks of String Geometries. Phys. Rev. Lett. 2018, 121, 101602. [CrossRef]

30. Denef, F. Les Houches Lectures on Constructing String Vacua. arXiv 2008, arXiv:0803.1194.

31. Silverstein, E. TASI lectures on cosmological observables and string theory. In New Frontiers in Fields and Strings; World Scientific: Singapore, 2016.

32. Berger, M. A Panoramic View of Riemannian Geometry; Springer: Berlin, Germany, 2003.

33. Douglas, M.R.; Kallosh, R. Compactification on negatively curved manifolds. J. High Energy Phys. 2010, 2010, 004 .

34. Candelas, P.; Perevalov, E.; Rajesh, G. Toric geometry and enhanced gauge symmetry of F theory/heterotic vacua. Nucl. Phys. B 1997, 507, 445-474. [CrossRef]

35. Banks, T.; Dixon, L.J. Constraints on String Vacua with Space-Time Supersymmetry. Nucl. Phys. B 1988, 307, 93-108. [CrossRef]

36. Arkani-Hamed, N.; Motl, L.; Nicolis, A.; Vafa, C. The String landscape, black holes and gravity as the weakest force. J. High Energy Phys. 2007, 2007, 60. [CrossRef]

37. Palti, E. The Swampland: Introduction and Review. Fortschritte Phys. 2019, 67, 1900037. [CrossRef]

38. Giddings, S.B.; Kachru, S.; Polchinski, J. Hierarchies from fluxes in string compactifications. Phys. Rev. D 2003, 66, 106006. [CrossRef]

39. Candelas, P.; De La Ossa, X.C.; Green, P.S.; Parkes, L. A Pair of Calabi-Yau manifolds as an exactly soluble superconformal theory. Nucl. Phys. B 1991, 359, 21-74. [AMS/IP Stud. Adv. Math. 1998, 9, 31-95.] [CrossRef]

40. Kachru, S.; Kallosh, R.; Linde, A.D.; Trivedi, S.P. De Sitter vacua in string theory. Phys. Rev. D 2003, 68, 046005. [CrossRef]

41. Balasubramanian, V.; Berglund, P.; Conlon, J.P.; Quevedo, F. Systematics of Moduli Stabilisation in Calabi-Yau Flux Compactifications. J. High Energy Phys. 2005, 2005, 7. [CrossRef]

42. Conlon, J.P.; Quevedo, F.; Suruliz, K. Large-volume flux compactifications: Moduli spectrum and D3/D7 soft supersymmetry breaking. J. High Energy Phys. 2005, 2005, 7. [CrossRef]

43. Becker, K.; Becker, M.; Haack, M.; Louis, J. Supersymmetry breaking and alpha-prime corrections to flux induced potentials. J. High Energy Phys. 2002, 2002, 60. [CrossRef]

44. Kachru, S.; Pearson, J.; Verlinde, H.L. Brane/flux annihilation and the string dual of a nonsupersymmetric field theory. J. High Energy Phys. 2002, 2002, 21. [CrossRef]

45. Marsh, D.; McAllister, L.; Wrase, T. The Wasteland of Random Supergravities. J. High Energy Phys. 2012, 2012, 102. [CrossRef]

46. Dine, M. Classical and Quantum Stability in Putative Landscapes. J. High Energy Phys. 2017, $2017,82$. 
[CrossRef]

47. Yamada, M.; Vilenkin, A. Hessian eigenvalue distribution in a random Gaussian landscape. J. High Energy Phys. 2018, 2018, 29. [CrossRef]

48. Banks, T.; Dine, M.; Gorbatov, E. Is there a string theory landscape? J. High Energy Phys. 2004, $2004,58$. [CrossRef]

49. Sethi, S. Supersymmetry Breaking by Fluxes. arXiv 2017, arXiv:1709.03554.

50. Danielsson, U.H.; Riet, T.V. What if string theory has no de Sitter vacua? Int. J. Mod. Phys. D 2018, $27,1830007$. [CrossRef]

51. Obied, G.; Ooguri, H.; Spodyneiko, L.; Vafa, C. De Sitter Space and the Swampland. arXiv 2018, arXiv:1806.08362.

52. Ooguri, H.; Palti, E.; Shiu, G.; Vafa, C. Distance and de Sitter Conjectures on the Swampland. arXiv 2018, arXiv:1810.05506.

53. Conlon, J.P. The de Sitter swampland conjecture and supersymmetric AdS vacua. arXiv 2018, arXiv:1808.05040.

54. Kachru, S.; Trivedi, S. A comment on effective field theories of flux vacua. arXiv 2018, arXiv:1808.08971.

55. Akrami, Y.; Kallosh, R.; Linde, A.; Vardanyan, V. The landscape, the swampland and the era of precision cosmology. arXiv 2018, arXiv:1808.09440.

56. Gibbons, G.W. Aspects Of Supergravity Theories. In Proceedings of the Three lectures Given at GIFT Seminar on Theoretical Physics, San Feliu de Guixols, Spain, 4-11 June 1984.

57. Gibbons, G.W. Thoughts on tachyon cosmology. Class. Quant. Grav. 2003, 20, S321. [CrossRef]

58. de Wit, B.; Smit, D.J.; Dass, N.D.H. Residual Supersymmetry of Compactified D = 10 Supergravity. Nucl. Phys. B 1987, 283, 165-191. [CrossRef]

59. Maldacena, J.M.; Nunez, C. Supergravity description of field theories on curved manifolds and a no go theorem. Int. J. Mod. Phys. A 2001, 16, 822. [CrossRef]

60. Moritz, J.; Retolaza, A.; Westphal, A. Toward de Sitter space from ten dimensions. Phys. Rev. D 2018, 97, 046010. [CrossRef]

61. Moritz, J.; Retolaza, A.; Westphal, A. On uplifts by warped anti-D3-branes. arXiv 2018, arXiv:1809.06618.

62. Bena, I.; Dudas, E.; Graña, M.; Lüst, S. Uplifting Runaways. arXiv 2018, arXiv:1809.06861.

63. Douglas, M.R. Landscape and Complexity Catastrophe. Talk Presented at the 2018 String_data Workshop in Munich. Available online: https://indico.mpp.mpg.de/event/5578/contribution/18/material/slides/0.pdf (accessed on 19 July 2019).

64. Guth, A.H. Eternal inflation and its implications. J. Phys. A Math. Theor. 2007, 40, 6811. [CrossRef]

65. Freivogel, B. Making predictions in the multiverse. Class. Quantum Gravity 2011, 28, 204007. [CrossRef]

66. Denef, F.; Douglas, M.R.; Greene, B.; Zukowski, C. Computational complexity of the landscape IICosmological considerations. Ann. Phys. 2018, 392, 93-127. [CrossRef]

67. Coleman, S.R.; Luccia, F.D. Gravitational Effects on and of Vacuum Decay. Phys. Rev. D 1980, $21,3305$. [CrossRef]

68. Denef, F.; Douglas, M.R. Computational complexity of the landscape I. Ann. Phys. 2007, 322, $1096-1142$. [CrossRef]

69. Dine, M.; Festuccia, G.; Morisse, A.; van den Broek, K. Metastable Domains of the Landscape. J. High Energy Phys. 2008, 2008, 14. [CrossRef]

70. Garriga, J.; Vilenkin, A. Watchers of the multiverse. J. High Energy Phys. 2013, 2013, 37. [CrossRef]

71. Dyson, L.; Kleban, M.; Susskind, L. Disturbing implications of a cosmological constant. J. High Energy Phys. 2002, 2002, 11. [CrossRef]

72. Albrecht, A.; Sorbo, L. Can the universe afford inflation? Phys. Rev. D 2004, 70, 063528. [CrossRef]

73. Brown, A.R.; Roberts, D.A.; Susskind, L.; Swingle, B.; Zhao, Y. Complexity, action, and black holes. Phys. Rev. D 2016, 93, 086006. [CrossRef]

74. Lloyd, S. Computational capacity of the universe. Phys. Rev. Lett. 2002, 88, 237901. [CrossRef]

(C) 2019 by the authors. Licensee MDPI, Basel, Switzerland. This article is an open access article distributed under the terms and conditions of the Creative Commons Attribution (CC BY) license (http:/ / creativecommons.org/licenses/by/4.0/). 